\title{
Permeabilización del Canal Radicular y Transporte Apical: Comparación de Tres Sistemas
}

\author{
Root Canal Glide Path and Apical Transportation: Three System Comparison
}

\author{
Francisca Muñoz'; Valeria Vargas²; Ximena Romero²; Silvia Vallejo² \& Raúl Alcántara1
}

MUÑOZ, F.; VARGAS, V.; ROMERO, X.; VALLEJO, S. \& ALCÁNTARA, R. Permeabilización del canal radicular y transporte apical: Comparación de tres sistemas. Int. J. Odontostomat., 11(2):151-156, 2017.

RESUMEN: La mantención de la anatomía original del canal radicular incide directamente en el éxito del tratamiento endodóntico. Para ello, los sistemas rotatorios de instrumentación requieren de canales radiculares permeables. Existen distintas limas y sistemas para la permeabilización o glide path como las limas tipo K manuales de acero inoxidable y los sistemas de NiTi rotatorios PathFile y ProGlider (Dentsply, Maillefer). Así, el objetivo de esta investigación fue comparar la capacidad de distintos sistemas de permeabilización para mantener la anatomía radicular sin producir transporte apical en canales mesiales de molares inferiores humanos extraídos. Se realizó un estudio cuantitativo experimental cuya muestra estuvo conformada por 36 canales mesiales de molares mandibulares humanos extraídos. Las muestras fueron divididas aleatoriamente en tres grupos conformados por 12 canales, cada uno de los cuales fueron sometidos a distintos sistemas de permeabilización (Lima K, PathFile y ProGlider). Las muestras fueron fotografiadas antes y después de la permeabilización utilizando un microscopio con magnificación 30X. Se cuantificó el desplazamiento del canal radicular en sentido mesio distal y buco - lingual posterior a la permeabilización. Para el análisis de los datos se utilizó el paquete estadístico InfoStat/ L y se aplicó la prueba de ANOVA / Tukey. Se observó que el sistema PathFile produce mayor transporte del canal radicular en su tercio apical en la dirección mesio-distal $(p=0,77)$ y el sistema ProGlider en la dirección buco-lingual ( $p=0,57)$. Sin embargo, estas diferencias no fueron significativas. En conclusión, los sistemas de permeabilización analizados no presentaron diferencias en cuanto a su capacidad para mantener la anatomía sin producir transporte apical.

PALABRAS CLAVE: permeabilización, transporte apical, instrumentación rotatoria, níquel titanio, pathfile, proglider.

\section{INTRODUCCIÓN}

La preparación biomecánica es una etapa crucial del tratamiento de endodoncia, sin embargo, no se encuentra exenta de inconvenientes. Uno de los principales problemas es la deformación innecesaria de los canales radiculares y la alteración de la morfología original del ápice radicular lo que da origen a perforaciones, transportes o formación de Zip. Así, la mantención de la anatomía original sin causar ningún evento iatrogénico, incide directamente en el éxito del tratamiento (Alves et al., 2012; Marzouk \& Ghoneim, 2013). La instrumentación mecanizada se ha desarrollado en las últimas décadas con el fin de evitar este tipo de accidentes ya que se ha caracterizado por su eficiencia en mantener la anatomía radicular interna (Ajuz et al., 2013; Marzouk \&
Ghoneim). Sin embargo, el uso de sistemas rotatorios, requiere que los canales radiculares se encuentren permeables.

La permeabilización en endodoncia, o glide path, ha sido descrita como la ejecución de una trayectoria inicial que permita el acceso a la entrada del canal radicular y el recorrido a través de toda su extensión en forma segura e ininterrumpida hasta el foramen apical (Kirchhoff et al., 2015). Este procedimiento, junto con la ampliación coronal del canal radicular, ha demostrado ser fundamental para un uso más seguro de los instrumentos de NiTi rotatorio, ya que impide que se produzcan aberraciones de forma y/o fracturas de los instrumentos (D'Amario et al., 2013).

\footnotetext{
${ }^{1}$ Departamento de Odontología Restauradora, Facultad de Odontología, Universidad de Concepción, Chile.

${ }^{2}$ Estudiante Programa de Especialización en Endodoncia 2015, Facultad de Odontología, Universidad de Concepción, Chile.

Proyecto financiado VRID n²15.102.019-1.0, Univesidad de Concepción, Chile.
} 
Al realizar el glide path se busca crear una forma inicial suave dentro del canal radicular, por lo general de una conicidad pequeña $(0,02)$ y de calibre 15 o 20 para evitar el bloqueo del instrumento rotatorio (Alovisi et al., 2017). Así, se logra que el diámetro inicial del canal radicular sea más amplio o al menos del mismo calibre que la punta del primer instrumento rotatorio utilizado (Berutti et al., 2009; Alves et al.). Para ello existen distintas limas y sistemas para la permeabilización o que "abren camino" para la instrumentación mecanizada las que deben producir un ensanchamiento inicial suave y centrado desde el origen del canal hasta el foramen apical (Ajuz et al.). Los llamados "instrumentos de permeabilización" facilitan tanto la ubicación del foramen como el acceso al tercio apical del canal radicular, permitiendo la creación de una exploración inicial y reduciendo al mínimo los errores de procedimiento durante el tratamiento endodóntico como son el transporte, zip y perforaciones (Türker \& Uzunoglu, 2015). Los instrumentos utilizados para la permeabilización del canal radicular deben ser idealmente de calibres pequeños y flexibles, pues deben permitir su progresión en dirección apical con seguridad y eficiencia, además de producir una ampliación inicial del canal suave y centrada en toda su extensión (Ajuz et al.).

Un tipo de instrumento de permeabilización son las limas tipo $\mathrm{K}$ manuales de acero inoxidable. Tradicionalmente, se ha propuesto el uso de estas limas para explorar el sistema de canales radiculares, lograr la patencia apical y proveer información adicional en relación a la forma tridimensional del canal, pues estos instrumentos mantienen la forma del canal una vez que son retirados (Bürklein et al., 2014). Comúnmente se utilizan limas $\mathrm{K}$ de diámetro pequeño precurvadas, en especial de calibres 6, 8, 10 y 15 .

Adicionalmente, en la actualidad, se han desarrollado variados sistemas de NiTi rotatorios con el propósito de permeabilizar. PathFile (Dentsply, Maillefer) es uno de los sistemas creados con estos fines, es fabricado en una aleación de NiTi austenitica convencional y consta de tres limas con conicidad 0,02 y de sección transversal cuadrada. En relación a este sistema existen tanto estudios que afirman que preservarían de mejor manera la anatomía original del canal radicular, como otros que indican que no existirían diferencias entre el uso de este sistema y el de limas $\mathrm{K}$ manuales (Berutti et al.; Alves et al.; Pasqualini et al., 2012; Alovisi et al.).

Otro sistema de permeabilización es ProGlider (Dentsply, Maillefer), su principal característica es que consta de un único instrumento en aleación NiTi MWIRE con punta calibre 16 y conicidad inicial de 0,02 , la que varía a lo largo del instrumento (Türker \& Uzunoglu; Kirchhoff et al.; Alovisi et al.). ProGlider, por ser un sistema relativamente nuevo, presenta pocos estudios en cuanto a su capacidad de conformación, sin embargo, publicaciones recientes describen que mantendría de mejor manera la anatomía apical del canal radicular al compararlo con la permeabilización con limas $\mathrm{K}$ manuales y/o con el sistema PathFile (Kirchhoff et al.; Alovisi et al.) Asimismo, se ha informado que tendría una flexibilidad significativamente más alta, además de una mayor resistencia a la fatiga cíclica y estrés torsional que PathFile (Elnaghy \& Elsaka, 2015).

A pesar de la importancia que tiene el procedimiento de glide path o permeabilización, existen pocas investigaciones que evalúen las desviaciones producidas en la trayectoria original del canal radicular por este tipo de instrumentos. Es por ello que el objetivo de esta investigación fue comparar la capacidad de distintos sistemas de permeabilización para mantener la anatomía radicular sin producir transporte apical en canales mesiales de molares inferiores humanos extraídos.

\section{MATERIAL Y MÉTODO}

Se realizó un estudio cuantitativo experimental cuya muestra estuvo conformada por canales mesiales de molares mandibulares humanos. Esto molares fueron extraídos por presencia de caries y/o enfermedad periodontal que impedía su rehabilitación y fueron recolectados en un Centro de Salud Familiar (CESFAM) chileno. Los molares fueron voluntariamente donados por pacientes sometidos a procedimientos de extracción cuya identidad es desconocida para todos los investigadores involucrados en el estudio. Además, el estudio contó con la autorización del Encargado del Programa Dental y el Director del CESFAM y fue aprobado por el Comité de Ética de la Facultad de Odontología de la Universidad de Concepción (C.Y.I.B. nº 01/16).

Se seleccionaron un total de 36 muestras para lo cual se incluyeron aquellos canales de raices mesiales con curvaturas radiculares moderadas $\left(10^{\circ}-24^{\circ}\right)$ de acuerdo al coeficiente de curvatura de Schneider (1971) cuya longitud medida desde el limite amelocementario hasta el domo apical era igual o superior a $11 \mathrm{~mm}$, que presentaban ápices maduros, con configuración radicular tipo III de Weine et al. (2012), cuyos canales mesiolinguales y mesiobucales estaban permeables y 
su calibre original era igual o menor a un instrumento ISO $n^{\circ} 10$. Se excluyeron aquellos canales de raíces: mesiales fusionadas con raíces distales, con reabsorción interna o externa, con canales calcificados, con caries radicular, con crack o fractura visibles y/o con tratamiento endodóntico previo.

Las coronas de cada diente fueron en primer lugar separadas a nivel del límite amelocementario y posteriormente se separó la raíz mesial de la distal. Luego, el acceso cervical y el foramen apical de los canales fueron sellados con cera roja y cada raíz fue sumergida en una solución de azul de metileno al $2 \%$ durante 10 segundos y luego secada con papel absorbente.

Posteriormente, se determinó la longitud de trabajo de cada canal radicular introduciendo una lima $\mathrm{K}$ calibre 10 (Denstply-Maillefer) observando la aparición de la lima a través del foramen apical y restándole a este valor $1 \mathrm{~mm}$.

A continuación, las raíces fueron montadas en cubos según la metodología descrita por Bramante (1987) y modificada más tarde por Kuttler et al. (2001), y se realizaron cortes a $4 \mathrm{~mm}$ del foramen apical con una máquina cortadora con sierra diamantada para separar el tercio apical de las muestras de manera de observar sólo este segmento de trabajo (Fig.1).

Para la permeabilización inicial de todos los canales se utilizaron limas $\mathrm{K}$ de acero inoxidable (Denstply-Maillefer) hasta calibre el \#10 irrigando con $1 \mathrm{ml}$ de solución de Hipoclorito de sodio al 2,5 \% llevándola al interior de los canales con una jeringa endodóntica Monoject (Kendall) de $3 \mathrm{ml}$ calibre $27 \mathrm{G}$

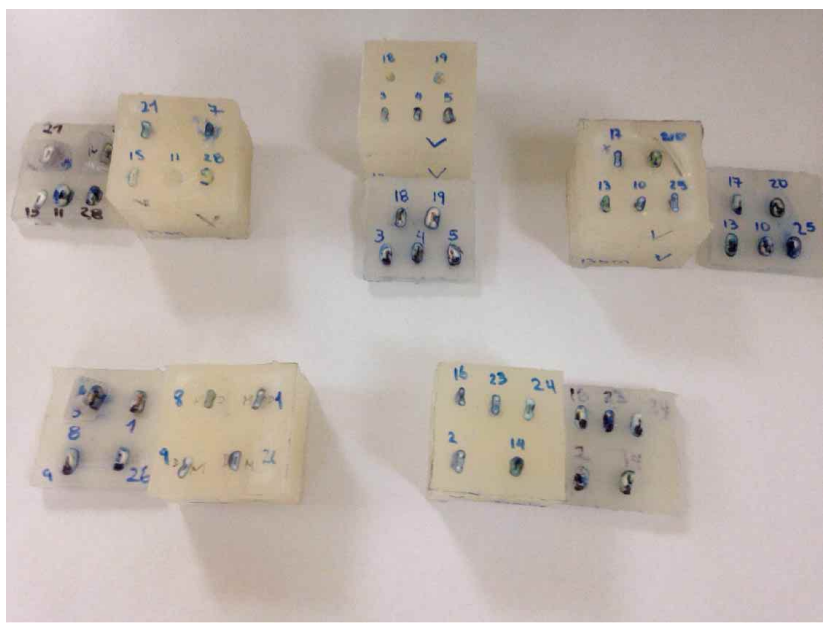

Fig. 1. Muestras montadas en cubos acrílicos de acuerdo a la metodología de Kuttler et al. (2001) y el corte de su tercio apical. ubicada dentro del conducto radicular hasta encontrar resistencia con un máximo de penetración de $3 \mathrm{~mm}$ hacia coronal de la longitud de trabajo. Finalmente, las muestras fueron divididas aleatoriamente en tres grupos conformados por 12 canales cada uno los que fueron sometidos a distintos sistemas de permeabilización utilizados por un sólo operador. En el grupo 1 se utilizaron limas K (Denstply-Maillefer) manuales (Calibres 008, 010, 015 y 020) manteniendo una irrigación con $1 \mathrm{ml}$ de Hipoclorito de sodio al 2,5\% entre cada instrumento. En el grupo 2 se utilizaron las limas rotatorias PathFile (Denstply-Maillefer) y en el grupo 3 la lima rotatoria ProGlider (Denstply-Maillefer), ambos sistemas de permeabillización mecanizada fueron utilizados en el motor X- Smart Plus (Dentsply, Maillefer) a una velocidad de 300 rpm y torque $2 \mathrm{Ncm}$, de acuerdo a lo recomendado por el fabricante. Asismismo, se utilizó glicerina como lubricante manteniendo la permeabilidad con lima K \#10 e irrigando con $1 \mathrm{ml}$ de Hipoclorito de sodio al $2,5 \%$ entre cada uno de los instrumentos. Una vez terminada la instrumentación, todos los canales fueron secados con conos de papel.

Las muestras fueron fotografiadas antes y después de la permeabilización utilizando un microscopio (PRIMA ENT/DNT, Labomed) con magnificación 30X. Luego mediante el uso del programa de edición fotográfica PhotoScape (MOOII Tech, Corea) las imágenes fueron sometidas a la aplicación de escala de grises en su formato blanco y negro y luego comparadas utilizando el programa ImageJ (NHI), previamente calibrado, cuantificándose el desplazamiento del canal radicular en sentido mesio - distal y buco - lingual posterior a la permeabilización, lo cual fue expresado en $\mathrm{mm}$ (Fig. 2).

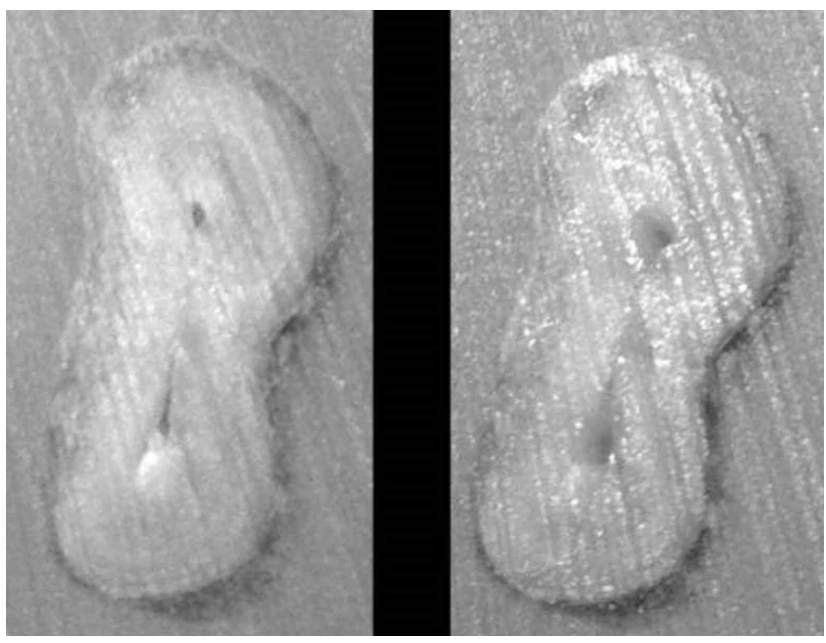

Fig. 2. Muestras fotografiadas antes y después de la permeabilización. 
Para el análisis de los datos se utilizó el paquete estadístico InfoStat/L (Versión 2015), se determinaron los estadísticos descriptivos por cada variable dependiente y se aplicó la prueba de ANOVA / Tukey con un intervalo de confianza del $95 \%$.

\section{RESULTADOS}

La Tabla I resume las medidas de tendencia central que describen el desplazamiento M-D originado por cada sistema de permeabilización, en ella se puede observar una mayor media para el desplazamiento producido por las limas PathFile $(0,12 \mathrm{~mm})$. La Tabla II resume las medidas de tendencia central que describen el desplazamiento B-L originado por cada sistema de permeabilización, en ella se puede observar una mayor media para el desplazamiento producido por las limas ProGlider $(0,27 \mathrm{~mm})$. Para todos los sistemas y tanto para el desplazamiento M-D del canal radicular como B-L cabe mencionar que la desviación estándar fue alta.

Tabla I. Estadísticos descriptivos del desplazamiento M-D de los conductos radiculares luego de la permeabilización con limas K-File, PathFile y Proglider. $(n=36)$

\begin{tabular}{llllll}
\hline Instrumento & $n$ & $\begin{array}{c}\text { Media } \\
(\mathrm{mm})\end{array}$ & D.E. & Mín & Máx \\
\hline K-File & 12 & 0,10 & 0,09 & 0,01 & 0,31 \\
PathFile & 12 & 0,12 & 0,10 & 0,00 & 0,38 \\
ProGlide & 12 & 0,09 & 0,09 & 0,01 & 0,29 \\
\hline
\end{tabular}

Al comparar los desplazamientos producidos por cada tipo de instrumento, las limas PathFile produjeron un mayor transporte del canal radicular en sentido M-D ( $p=0,771)$ Fig. 3) y las limas ProGlider en sentido B-L $(0,57)$ (Fig. 4). Sin embargo, ninguna de estas diferencias fueron significativas.

Tabla II. Estadísticos descriptivos del desplazamiento B-L de los conductos radiculares luego de la permeabilización con limas K-File, PathFile y Proglider.

\begin{tabular}{lcllll}
\hline Instrumento & $\mathrm{n}$ & $\begin{array}{l}\text { Media } \\
(\mathrm{mm})\end{array}$ & D.E. & Mín & Máx \\
\hline K-File & 12 & 0,22 & 0,19 & 0,00 & 0,52 \\
PathFile & 12 & 0,19 & 0,19 & 0,00 & 0,66 \\
ProGlide & 12 & 0,27 & 0,18 & 0,00 & 0,64 \\
\hline
\end{tabular}

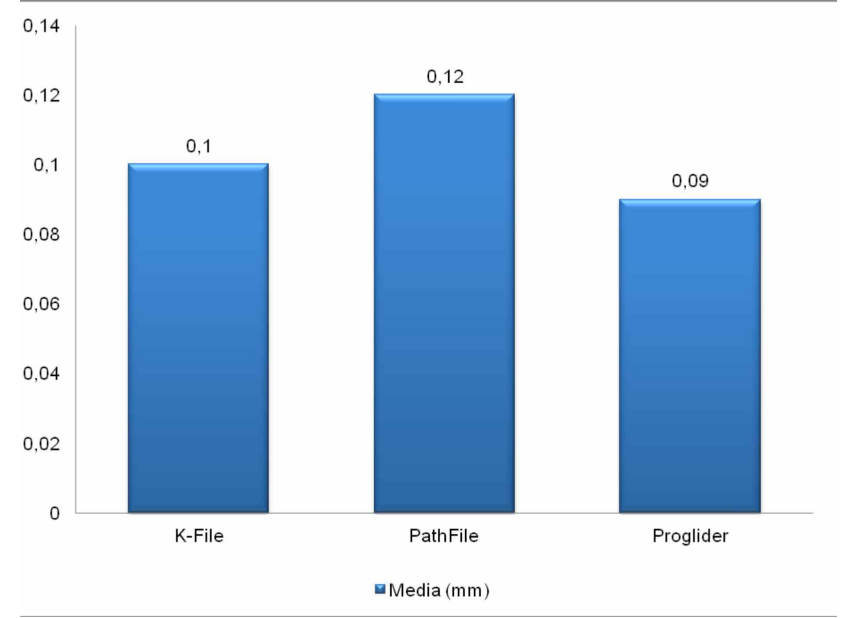

Fig.3. Comparación del desplazamiento del conducto radicular en sentido $M-D$ luego de la permeabilización con limas K-File, PathFile y ProGlider $(n=36 ; p=0,77)$

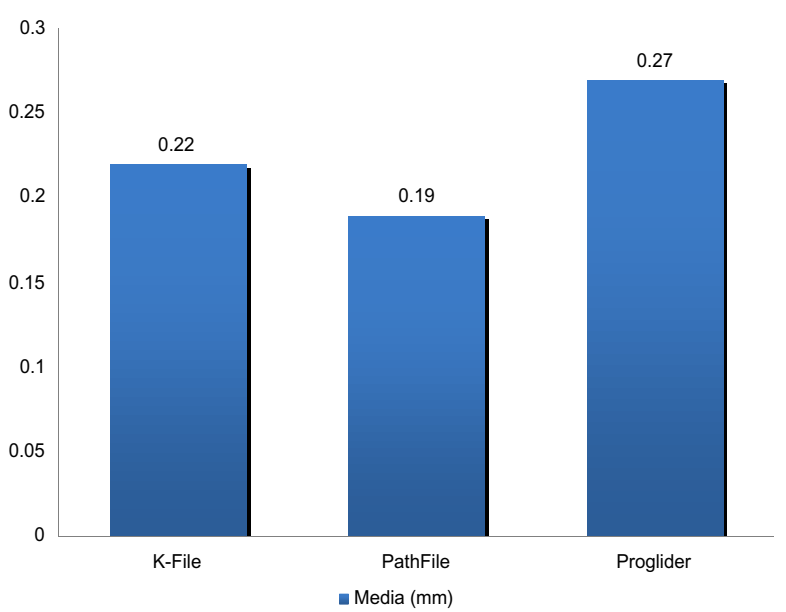

Fig.4. Comparación del desplazamiento del conducto radicular en sentido B-L luego de la permeabilización con limas K-File, PathFile y ProGlider $(n=36 ; p=0,57)$.

\section{DISCUSIÓN}

Los factores que podrían generar transporte del canal radicular han sido ampliamente estudiados debido a que aumentan el riesgo de bloqueo, perforación, escalones y eliminación innecesaria de la estructura dentaria, lo que puede resultar en un pronóstico desfavorable del tratamiento endodóntico (Kirchhoff et al.). Dentro de ellos, el establecimiento de la permeabilidad o glide path es esencial para permitir el desempeño adecuado de los instrumentos de NiTi rotatorios sin producir transporte (Alovisi et al.). 
El presente estudio tuvo por objetivo evaluar sistemas de permeabilización, tanto manuales como rotatorios, en canales curvos y finos que representan un mayor desafío clínico. Los resultados indican que tanto la utilización de limas $\mathrm{K}$ manuales como de los sistemas rotarios PathFile y ProGlider permiten mantener de igual forma la anatomía apical del canal radicular durante la permeabilización. Estos resultados son similares a lo observado en los trabajos de Kirchhoff et al. y Alovisi et al., quienes estudiaron los mismos sistemas investigados sin observar diferencias significativas en el transporte apical al utilizar ProGlider y PathFile. Sin embargo, Alovisi et al. en su investigación encontraron que ambas limas rotatorias permitían mantener de mejor manera la anatomía apical que las limas K. En relación a ello, otros autores como Berutti et al. y Pasqualini et al. reportaron una menor ocurrencia de transporte apical y deformación del canal radicular en canales permeabilizados con instrumentos rotatorios PathFile cuando fueron comparados con canales instrumentados con limas $\mathrm{K}$ manuales. Por otro lado, Alves et al. y D'Amario et al. observaron que no existe influencia alguna en el transporte apical o aberración del canal al comparar el uso del sistema PathFile con limas $\mathrm{K}$ manuales. Sus resultados que son similares a los de Türker \& Uzunoglu, quienes observaron la presencia de transporte apical luego de finalizar la instrumentación con limas mecanizadas Protaper Next en canales permeabilizados con los tres sistemas estudiados en esta investigación, no observando diferencias significativas entre ellos. A raíz de los diversos resultados encontrados en la literatura incluyendo este estudio, es que por el momento tanto las técnicas manuales como rotatorias son clínicamente aceptadas para las realización de la permeabilización o glide path (Alovisi et al.).

A pesar de ello, cabe destacar que existen otras diferencias entre ambos sistemas que también son relevantes para la clínica. En este sentido, Kirchhoff et al. destacaron que ProGlider al ser una lima única permite establecer permeabilidad en forma más rápida que PathFile. Además, otros estudios han destacado las ventajas respecto al comportamiento mecánico de Proglider por sobre Pathfile (Elnaghy \& Elsaka).

Uno de los principales factores que podría contribuir al resultado obtenido en este estudio y la inconstancia de los resultados observados en la literatura es la alta variabilidad anatómica del canal radicular. Esta variabilidad es particularmente importante en la porción apical del canal radicular y podría tener un alto impacto en la habilidad de mantener patencia y un glide path reproducible como ha sido planteado por Min et al. (2016). Por ello, a pesar que las muestras fueron cuidadosamente seleccionadas y se utilizaron dientes naturales, estos suelen ser difíciles de estandarizar. A raíz de ello, sugerimos que se realicen más estudios que evalúen la capacidad de mantener la anatomía radicular en canales de una mayor variedad de dientes y con los distintos sistemas disponibles en el mercado.

Finalmente, concluimos que los sistemas de permeabilización analizados no presentaron diferencias en cuanto a su capacidad de mantención de la anatomía sin producir transporte apical. Así, con la información existente hasta el momento, cualquiera de ellos pudiera ser utilizado de forma segura para la permeabilización del canal radicular.

MUÑOZ, F.; VARGAS, V.; ROMERO, X.; VALLEJO, S. \& ALCÁNTARA, R. Root canal glide path and apical transportation: three system comparison. Int. J. Odontostomat., 11(2):151-156, 2017.

ABSTRACT: Preservation of the original root canal anatomy has a direct influence on the success of an endodontic treatment. In order to achieve this, rotary instrumentation systems require permeable root canals. Different files and systems are used for the establishment of a glide path such as manual stainless steel $\mathrm{K}$ files and NiTi rotatory systems like PathFile and ProGlider (Dentsply, Maillefer). Thus, the objective of this research was to compare the ability of different systems to create a glide path and maintain root canal anatomy without producing apical transportation in mesial root canals of extracted human lower molars. A quantitative experimental study was performed with a sample of 36 mesial root canals of extracted human mandibular molars. The samples were randomly divided into three groups consisting of 12 root canals each, which were subjected to different glide path systems (K-Files, PathFile and ProGlider). Samples were photographed before and after creating glide path using a microscope with $30 \mathrm{X}$ magnification. The displacement of the root canal in a mesio - distal and bucco - lingual direction was quantified after creating glide path. Data was analyzed using the statistical package InfoStat / $L$ and the ANOVA / Tukey test was applied. The PathFile system produced greater transport of the root canal in its apical third in the mesiodistal direction $(p=0.77)$ and the ProGlider system in the bucco-lingual direction $(p=0.57)$. However, these 
differences were not significant. In conclusion, the glide path systems analyzed do not present any differences in their ability to maintain the anatomy without producing apical transportation.

\section{KEY WORDS: glide path, apical transportation, rotary instrumentation, nickel- titanium, pathfile, proglider.}

\section{REFERENCIAS BIBLIOGRÁFICAS}

Ajuz, N. C.; Armada, L.; Gonçalves, L. S.; Debelian, G. \& Siqueira, J. F. Jr. Glide path preparation in S-shaped canals with rotary pathfinding nickel-titanium instruments. J. Endod., 39(4):534-7, 2013.

Alovisi, M.; Cemenasco, A.; Mancini, L.; Paolino, D.; Scotti, N.; Bianchi, C. C. \& Pasqualini, D. Micro-CT evaluation of several glide path techniques and ProTaper Next shaping outcomes in maxillary first molar curved canals. Int. Endod. J., 50(4):387-97, 2017.

Alves, V. de O.; Bueno, C. E.; Cunha, R. S.; Pinheiro, S. L.; Fontana, C. E. \& de Martin, A.S. Comparison among manual instruments and PathFile and Mtwo rotary instruments to create a glide path in the root canal preparation of curved canals. $J$. Endod., 38(1):117-20, 2012.

Berutti, E.; Cantatore, G.; Castellucci, A.; Chiandussi, G.; Pera, F.; Migliaretti, G. \& Pasqualini, D. Use of nickel-titanium rotary PathFile to create the glide path: comparison with manual preflaring in simulated root canals. J. Endod., 35(3):408-12, 2009.

Bürklein, S.; Poschmann, T. \& Schäfer, E. Shaping ability of different nickel-titanium systems in simulated S-shaped canals with and without glide path. J. Endod., 40(8):1231-4, 2014.

D'Amario, M.; Baldi, M.; Petricca, R.; De Angelis, F.; El Abed, R. \& D'Arcangelo, C. Evaluation of a new nickel-titanium system to create the glide path in root canal preparation of curved canals. J. Endod., 39(12):1581-4, 2013.

Elnaghy, A. M. \& Elsaka, S. E. Evaluation of the mechanical behaviour of PathFile and ProGlider pathfinding nickel-titanium rotary instruments. Int. Endod. J., 48(9):894-901, 2015.

Kirchhoff, A. L.; Chu, R.; Mello, I.; Garzon, A. D.; dos Santos, M. \& Cunha, R. S. Glide path management with single- and multipleinstrument rotary systems in curved canals: A micro-computed tomographic study. J. Endod., 41(11):1880-3, 2015.

Kuttler, S.; Garala, M.; Perez, R. \& Dorn, S. O. The endodontic cube: a system designed for evaluation of root canal anatomy and canal preparation. J. Endod., 27(8):533-6, 2001.

Marzouk, A. M. \& Ghoneim, A. G. Computed tomographic evaluation of canal shape instrumented by different kinematics rotary nickeltitanium systems. J. Endod., 39(7):906-9, 2013.

Min, Y.; Ma, J. Z.; Shen, Y.; Cheung, G. S. \& Gao, Y. A micro-computed tomography study of the negotiation and anatomical feature in apical root canal of mandibular molars. Scanning, 38(6):819-24, 2016.

Pasqualini, D.; Bianchi, C. C.; Paolino, D. S.; Mancini, L.; Cemenasco, A.; Cantatore, G.; Castellucci, A. \& Berutti, E. Computed microtomographic evaluation of glide path with nickel-titanium rotary PathFile in maxillary first molars curved canals. J. Endod., 38(3):389-93, 2012.

Schneider, S. W. A comparison of canal preparations in straight and curved root canals. Oral Surg. Oral Med. Oral Pathol., 32(2):2715,1971 .

156
Türker, S. A. \& Uzunog`lu, E. Apical root canal transportation of different pathfinding systems and their effects on shaping ability of ProTaper Next. J. Clin. Exp. Dent., 7(3):e392-5, 2015.

Weine, F. S.; Healey, H. J.; Gerstein, H. \& Evanson, L. Canal configuration in the mesiobuccal root of the maxillary first molar and its endodontic significance. 1969. J. Endod., 38(10):1305-8, 2012.

Dirección para correspondencia:

Dra. Francisca Muñoz Campos

Cirujano Dentista

Especialista en Endodoncia

Magister en Educación Médica

para las Ciencias de la Salud

Profesor Asistente

Facultad de Odontología

Universidad de Concepción

Concepción

CHILE

Email:francmunoz@udec.cl

Recibido : 14-11-2016

Aceptado: 01-03-2017 